\title{
7 Einblicke in die Studien- und Berufspraxis
}

\author{
Elfriede Wagner ${ }^{a}$ und Simon Huber ${ }^{b}$ \\ a Stabstelle Qualitätsmanagement (BOKU), ${ }^{b}$ UBRM-Alumni \\ elfriede.wagner@boku.ac.at, ubrm-alumni@boku.ac.at
}

\subsection{Ergebnisse aus Studien zu Absolventinnen und Absolventen}

Die BOKU untersucht seit 2013 regelmäßig den Arbeitsmarkt- und Berufserfolg ihrer Absolventinnen und Absolventen (BOKU 2019). Man weiß somit in etwa, welchen Berufsweg Absolventinnen und Absolventen der UBRM-Studien nach ihrem Abschluss einschlagen und wie erfolgreich sie dabei sind.

Der Großteil der UBRM-Bachelorabsolventinnen und -absolventen studiert weiter, die UBRM-Masterstudierenden drängen hingegen nach ihrem Abschluss auf den Arbeitsmarkt. UBRM-Diplomingenieurinnen und -ingenieure benötigen häufig eine etwas längere Such- und Orientierungsphase am Arbeitsmarkt. Jedoch steigt die leicht unterdurchschnittliche Erwerbsbeteiligung nach einem halben Jahr kontinuierlich an. Zwei Jahre nach dem Abschluss liegt sie bereits über dem BOKU-Durchschnitt. Insbesondere die Frauenerwerbsquoten sind bei UBRM-Absolventinnen und -Absolventen sehr hoch. Darüber hinaus zeigen sich überdurchschnittlich stabile Beschäftigungsverhältnisse.

UBRM-Absolventinnen und -absolventen arbeiten aufgrund ihrer vielseitigen Ausbildung in einer Vielzahl von Sektoren. Drei Jahre nach ihrem Abschluss sind sie am häufigsten in folgenden Branchen ${ }^{1}$ zu finden:

- öffentliche Verwaltung,

- Interessenvertretungen und Vereine,

- Erziehung und Unterricht (u.a. Universitäten),

- Architektur- und Ingenieurbüros,

- Abfallbehandlung,

- Unternehmensführung und -beratung,

- Energieversorgung,

- Großhandel und Einzelhandel.

Vergleichsweise wenig UBRM-Alumni gehen ins Ausland.

1 Die Gliederung erfolgt nach der ÖNACE-Klassifikation der Arbeitsstätte (siehe Statistik Austria 2018). 
Wie zufrieden sind UBRM-Absolventinnen und -absolventen nun im Beruf? Die KOAB-AbsolventInnenbefragung (BOKU 2017) zeigt, dass UBRM-Masterabsolventinnen und -absolventen (im Vergleich zu BOKU-Absolventinnen und -absolventen anderer Fächer) weniger studienfachnah beschäftigt sind, sie ihre Beschäftigung jedoch überdurchschnittlich oft an ihr Ausbildungsniveau angemessen einschätzen. Darüber hinaus sind sie - im BOKU-Vergleich - mit ihrer beruflichen Situation insgesamt zufriedener. UBRM-Bachelorabsolventinnen und -absolventen bewerten ihre berufliche Situation deutlich negativer: Sie sind öfter teilzeitbeschäftigt, können ihre im Studium erworbenen Qualifikationen weniger einsetzen, übernehmen seltener studienfachnahe berufliche Aufgaben und sind mit ihrer beruflichen Situation insgesamt unzufriedener als Absolventinnen und Absolventen anderer BOKU-Bachelorstudien.

\subsection{Porträts von UBRM-Absolventinnen und -Absolventen}

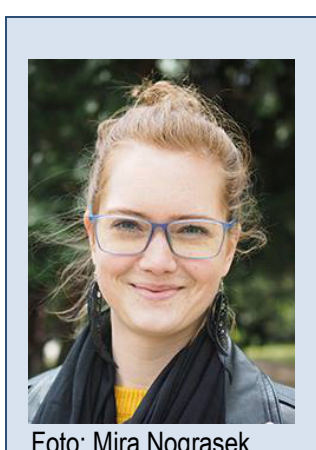

Foto: Mira Nograsek

\section{Elena Beringer, BSc}

UBRM-Bachelor (Abschluss 2016, BSc); UBRM-Master (laufend)

Aktuelle Tätigkeit: Selbstständige Trainerin

\section{Was ist UBRM für dich?}

UBRM ist für mich eine Verbindung verschiedener Sichtweisen auf unsere komplexe Welt und eine Studienrichtung, die einem die Möglichkeiten gibt, die Probleme unserer Zeit zu bearbeiten und zu verstehen. Die Interdisziplinarität und die Vielseitigkeit sind für mich die Stärken des Studiums, da man dadurch zwischen verschiedenen Personen und Positionen vermitteln lernt. Gleichzeitig ist UBRM für mich auch ein Studium, das meinen Horizont maßgeblich erweitert und reflektiertes Handeln und Denken stark gefördert hat, auch durch den häufigen Austausch mit den Studierenden untereinander.

\section{Was machst du in deinem Job?}

Als Trainerin arbeite ich interaktiv mit Gruppen zu unterschiedlichsten Themen. Teambuilding, Projektund Visionsarbeit, Kommunikation und Konfliktmanagement sind hier Schwerpunkte. Durch einen spielerischen Zugang und mit Gespür für die jeweilige Situation passe ich mich flexibel den Gegebenheiten in einem Seminar oder Workshop an. Inhaltlich arbeite ich auch in den Bereichen Klima, Nachhaltigkeit und Finanzkompetenz.

\section{Was hat dir UBRM dafür gebracht?}

Durch das Studium habe ich ausreichend Grundlagenwissen in den Bereichen Klima, Energie, Regionalentwicklung und Nachhaltigkeit vermittelt bekommen, was mir hilft, die Hintergründe meiner Tätigkeiten schnell zu erfassen. Ich kann mich so also auch inhaltlich besser auf die Gruppen einstellen. Kommunikationsfähigkeit, wissenschaftliches Arbeiten und Projektmanagement sind außerdem zentrale Fähigkeiten, die ich in meinem Beruf häufig brauche. Wichtig ist zudem die Fähigkeit, Inhalte zu reflektieren, kreative Ideen einbringen zu können und Zusammenhänge rasch zu verstehen. Der interdisziplinäre Zugang und ein Denken in Systemen sind ebenfalls besonders wertvoll. 


\section{Was empfiehlst du UBRM-Studierenden?}

Ich würde den Studierenden empfehlen, sich im Studium einerseits durch Wahlfächer zu spezialisieren und andererseits sich durch ehrenamtliches Engagement, zusätzliche Ausbildungen und Praktika persönlich weiterzuentwickeln. Viele Fertigkeiten, die mir in meinem Job zugutekommen, habe ich mir in anderweitigen Tätigkeiten angeeignet. Inspiration durch andere Studierende und ein Ideenaustausch auf diversen Veranstaltungen sind auch gute Wissens- und Austauschquellen für eine umfassende Qualifikation. Das Aufbauen eines Netzwerks ist hier ein weiterer Vorteil. Generell würde ich sagen: Engagiert euch für das, was euch wichtig ist!

Dipl.-Ing. Daniel Böhm, BSc
UBRM-Bachelor (Abschluss 2012, BSc); Master in Agrar- und Ernährungs-
wirtschaft (Abschluss 2016, Dipl.-Ing.)
Aktuelle Tätigkeit: Mitarbeiter im Event- und Projektmanagement sowie
Wür Social Media, Mobilitätsagentur Wien
UBRM ist für mich viel mehr als nur eine Studienrichtung. Es ist ein Lebens-
gefühl und eine Herangehensweise an gesellschaftliche und berufliche Herausforderungen. Die Vielfalt
der Studienfächer regt stets zur kritischen Diskussion an und ermöglicht eine differenzierte Betrach-
tung der Themen. UBRM hat mich gelehrt, interdisziplinär zu denken und komplexe Zusammenhänge
leichter zu verstehen.
Was machst du in deinem Job?
Ich bin für die Mobilitätsagentur Wien zuständig und betreue dort zahlreiche Projekte, die Menschen
zur aktiven Mobilität in der Stadt anregen sollen. Ich unterstütze das städtische Unternehmen dabei,
die Marken „Fahrrad Wien“ und „Wien zu Fuß“ zu kommunizieren und diese der Öffentlichkeit über
zahlreiche Kanäle und Veranstaltungen zu präsentieren. Zurzeit organisiere ich außerdem eine inter-
nationale Fachkonferenz.
Was hat dir UBRM dafür gebracht?
UBRM hat mir dabei geholfen, den interdisziplinären Herausforderungen der Arbeitswelt begegnen
zu können. Es hat mich gelehrt, gemeinsam mit anderen im Team an Projekten arbeiten zu können
und diese zu koordinieren. UBRM hat mir außerdem dabei geholfen, komplexe Themen verständlich
an die Öffentlichkeit kommunizieren zu können und so eine Übersetzerrolle einzunehmen.
Was empfiehlst du UBRM-Studierenden?
Ich empfehle den Studierenden, neugierig zu sein und sich Wissen aus möglichst vielen Bereichen
anzueignen. Dennoch sollten sie nicht ihre Talente und Interessen vergessen, sondern das Studium zur
Vertiefung dieser nutzen. Außerdem empfehle ich dringendst, die Studienzeit an der schönen BOKU
zu genießen, zu reisen und sich neben dem Studium so gut es geht zu engagieren.




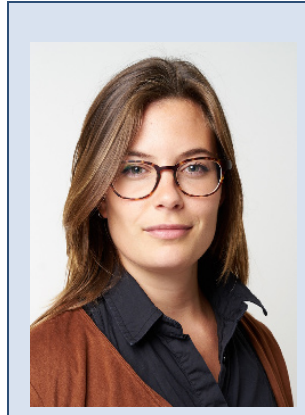

\section{Christina Trapl, MA BSc}

UBRM-Bachelor (Abschluss 2013, BSc); Professional Master in Public Communication, Spezialisierung Public Affairs (Abschluss 2016, MA); UBRMMaster, Spezialisierung Energie und Wasser (laufend)

Aktuelle Tätigkeit: Consultant and Public Affairs Expert, Grayling Austria GmbH

\section{Was ist UBRM für dich?}

UBRM ist für mich eine Plattform für Dialog und Menschen, die nachfragen und Dinge nicht als gegeben ansehen.

\section{Was machst du in deinem Job?}

Als Public-Relations- und Public-Affairs-Expertin berate ich Kundinnen und Kunden aus dem öffentlichen und privaten Sektor mit strategischer Kommunikation. Was heißt das genau? Ich bin Ideenpool, Themendetektivin, Strategin und Troubleshooterin in einer Person. In meiner täglichen Arbeit muss ich Problemstellungen kreativ lösen, wissen, mit wem man spricht, und pain points für meine Kundinnen und Kunden schnell erkennen können.

\section{Was hat dir UBRM dafür gebracht?}

Als Beraterin für große und kleine Unternehmen und Organisationen lese ich mich schnell in Themen ein, interessiere mich für Trends und gehe proaktiv auf Personen und Gruppen - mit unterschiedlichen Ideologien und Interessen - zu. Bei UBRM trifft Ökonomie auf Ökologie, jeder redet mit und hat die Chance, verschiedene wirtschafts- und gesellschaftspolitische Themen aus neuen Blickwinkeln zu betrachten. UBRM hat mich gelehrt, immer neugierig und aufgeschlossen an die Dinge heranzugehen.

\section{Was empfiehlst du UBRM-Studierenden?}

Manche kritisieren, dass UBRM zu oberflächlich ist. Mein Zugang dazu: Nutze das breite Studienangebot als Chance, um herauszufinden, was dich bewegt. Führe Gespräche mit Studierenden sowie mit Professorinnen und Professoren und fang früh an, dich auf ein Thema zu spezialisieren. So habe ich auch meinen ersten berufsspezifischen Job über die Kontakte meiner BOKU-Professorin bekommen.

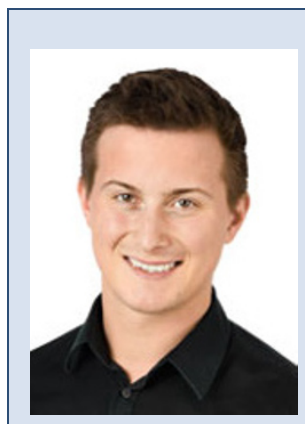

\section{Dipl.-Ing. Georg Weber, BSc}

Bachelor in Agrarwissenschaften (Abschluss 2013, BSc); UBRM-Master (Abschluss 2019, Dipl.-Ing.)

Aktuelle Tätigkeit: Junior Rohstoffkoordinator, Ja! Natürlich Naturprodukte Gesellschaft m.b.H.

\section{Was ist UBRM für dich?}

Für mich ist UBRM ein unglaublich vielseitiges Studium, das einem im Bachelor das breite Feld der Umwelt- und Nachhaltigkeitsthemen eröffnet und im Master die Möglichkeit bietet, sich ganz nach den individuellen Interessen zu vertiefen. Der Mix aus Natur-, Sozial- und Wirtschaftswissenschaften schafft in einer Vielzahl von Bereichen ein hervorragendes Basiswissen, auf das man im späteren Berufsleben aufbauen kann. 


\section{Was machst du in deinem Job?}

Im Ja! Natürlich-Team der Eigenmarkenabteilung der REWE International AG teilt sich mein Aufgabenfeld grob in zwei Bereiche. Einerseits handelt es sich dabei um ein aktives Rohstoffmanagement inklusive Bedarfskalkulationen und Entwicklungsbeobachtungen in den Bereichen Schwein, Wurst \& Schinken, Geflügel und Eier sowie in weiterer Folge auch Obst \& Gemüse. Andererseits widme ich mich der Analyse und Auswertung von Marktdaten zur Entwicklung der Bioanteile in allen Frischesegmenten im eigenen Haus wie auch beim Mitbewerb.

\section{Was hat dir UBRM dafür gebracht?}

Die umfassende, ganzheitliche Erfassung von Problemstellungen mit allen relevanten Akteurinnen und Akteuren und Faktoren sowie die Ableitung von Maßnahmen unter Berücksichtigung der Auswirkungen dieser ist ganz klar ein Learning, das ich aus dem UBRM-Studium mitnehmen konnte. Das analytische Denken und Finden von Lösungsansätzen, wie es in vielen Übungen im Studium gefordert wird, ist ganz klar ein wichtiger Bestandteil meiner Arbeit.

\section{Was empfiehlst du UBRM-Studierenden?}

Nutzt unbedingt das vielfältige Angebot an Veranstaltungen zu Themen eures Interesses an der BOKU und in Wien überhaupt. Wenn man das Studium lediglich auf das Lernen zu Hause oder in der Bibliothek beschränkt, entgehen einem meiner Meinung nach wertvoller, unterschiedlichster Input, spannende Diskussionen, unerwartete Möglichkeiten und glückliche Zufälle.

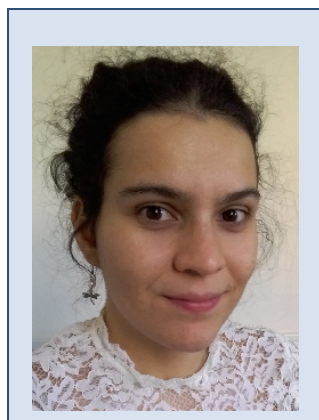

\section{Dipl.-Ing. Katharina Gruber, BSc \\ UBRM-Bachelor und -Master (Abschluss 2017, Dipl.-Ing.)}

Aktuelle Tätigkeit: Wissenschaftliche Projektmitarbeiterin am Institut für Nachhaltige Wirtschaftsentwicklung, BOKU

\section{Was ist UBRM für dich?}

Nach einem Jahr Elektrotechnikstudium, das für mich sehr theoretisch war, wollte ich etwas Vielseitigeres mit mehr Bezug zur Praxis machen, das auch, aber nicht nur, technische Themen umfasst. UBRM deckt mit einer Mischung aus technischen, wirtschaftlichen, sozial-, natur- und rechtswissenschaftlichen Themen ein breites Spektrum ab. Besonders schätze ich die inter- und transdisziplinäre Ausrichtung, welche es ermöglicht, die Schnittstellen verschiedener Wissenschaften wahrzunehmen und entsprechende ganzheitliche Systeme zu betrachten.

\section{Was machst du in deinem Job?}

Ich arbeite für das Forschungsprojekt reFUEL, das sich mit der Rolle des Handels erneuerbarer Energie in einer zukünftigen Welt befasst. Dabei beschäftige ich mich mit der Simulation von erneuerbarer Energieproduktion basierend auf Klimadaten und mit der Analyse dieser Zeitreihen. Auch Aspekte der Speicherung von Strom aus Erneuerbaren, Möglichkeiten des Transports und wirtschaftliche Belange werden dabei untersucht. Ich bin Teil eines Teams, das sich aus Expertinnen und Experten verschiedener Bereiche zusammensetzt, was Koordination und Kommunikation an diesen Schnittstellen notwendig macht.

Meine Hauptaufgaben bestehen darin, globale Klimadaten zu analysieren, wobei ich Modelle anwende und bewerte und diese Ergebnisse in schriftlicher oder mündlicher Form präsentiere. 


\section{Einblicke in die Studien- \& Berufspraxis}

\section{Was hat dir UBRM dafür gebracht?}

Das Know-how in verschiedenen wissenschaftlichen Bereichen ist für mich wichtig, da ich einerseits selbst mehrere Bereiche miteinander verbinde, insbesondere Klima und Energie. Andererseits ist ein breites Wissen auch notwendig, um über diverse Aspekte, die alle mit dem Umstieg auf erneuerbare Energiequellen zusammenhängen, diskutieren zu können - sowohl innerhalb des Projektteams als auch mit Expertinnen und Experten aus anderen Disziplinen, die oft wichtige Ansichten einbringen.

\section{Was empfiehlst du UBRM-Studierenden?}

Oft wird die Ansicht vertreten, UBRM wäre zu „oberflächlich“. Das mag daran liegen, dass das Bachelorstudium sehr breit gefächert ist. Detaillierteres Fachwissen für spezielle Tätigkeiten könnt inr beispielsweise im Rahmen von Praktika oder der Masterarbeit erwerben oder auch zu Beginn eurer beruflichen Tätigkeit.

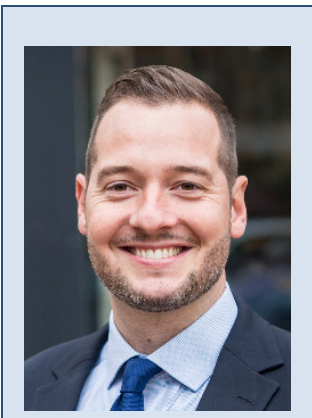

\section{Markus Ginders, BSc}

UBRM-Bachelor (Abschluss 2017, BSc)

Aktuelle Tätigkeit: Gründer von $\mathrm{CO} 2 m p e n s i o$ (weltweit erste App zur $\mathrm{CO}_{2-}$ Kompensation von Flugreisen und Autofahrten)

\section{Was ist UBRM für dich?}

Studieren war für mich nie ein Muss, sondern eher ein Luxus, den ich mir erst verdienen und dann gönnen wollte. Als ich mich für das UBRM-Studium entschied, war ich als Prokurist für ein deutsches Handelshaus in Senegal tätig und wollte eine Richtungsänderung in meiner Karriere. Das UBRM-Studium hat mich in meiner damaligen Lebenssituation ideal unterstützt, um mich im Bereich Nachhaltigkeit weiterzuentwickeln.

\section{Was machst du in deinem Job?}

Als Gründer von CO2mpensio habe ich die Idee \& Strategie entwickelt und kümmere mich um Partnerschaften mit Unternehmen und Kompensationspartnern wie dem Zentrum für Globalen Wandel \& Nachhaltigkeit an der BOKU. Ich bin Schnittstelle zwischen IT und Kundinnen und Kunden, Vertriebsleiter, mache die Öffentlichkeitsarbeit und betreue ein kleines, aber feines Team.

\section{Was hat dir UBRM dafür gebracht?}

Das UBRM-Studium hat mir einen guten Einblick in viele Aspekte der Ressourcenökonomie und Nachhaltigkeit gegeben. V.a. das fächerübergreifende Denken und das kollegiale Miteinander hilft mir fast täglich, einen guten Job zu machen.

\section{Was empfiehlst du UBRM-Studierenden?}

Rennt nicht durch eure Studienzeit, indem ihr Fragenkataloge auswendig lernt, sondern nutzt diese Phase eures Lebens, um das Denken neu zu entdecken. Macht ein Erasmus-Praktikum mit echtem Studienbezug. Nutzt gesellige Momente, um Theorien und Denkansätze zu diskutieren. Vergesst bei all dem Trubel nicht, dass regelmäßiger Schlaf und Bewegung helfen können, Gedanken und Ideen neu zu ordnen. Seid proaktiv in eurem Auftreten und behaltet euch eine positive Grundeinstellung zum Leben, auch wenn das Studium und die Menschen desillusionieren können. 


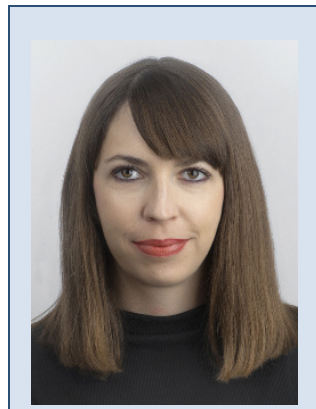

Dipl.-Ing. Carmen Schmid, BSc

UBRM-Bachelor und -Master (Abschluss 2013, Dipl.-Ing.)

Aktuelle Tätigkeit: Expertin für Treibhausgasinventuren und -projektionen, Umweltbundesamt

\section{Was ist UBRM für dich?}

Ich habe mich schon immer für ein Studium mit Schwerpunkt Umweltschutz interessiert, und ich habe mich bereits mit 16 Jahren entschieden, UBRM auf der BOKU zu studieren (zu der Zeit wurde das Studium gerade eingeführt). UBRM hat mir beigebracht, in verschiedenen Disziplinen zu denken und diese zu verstehen. Als UBRM-Absolventin bzW. -Absolvent besitzt man sehr viel implizites Wissen und spricht viele Fachsprachen. Diese Skills werden einem aber oft erst später im Berufsleben bewusst.

\section{Was machst du in deinem Job?}

Ich arbeite im Umweltbundesamt in der Abteilung für Klimaschutz und Emissionsinventuren. Ich bin einerseits für die Erstellung der österreichischen Treibhausgasinventur des Landnutzungs- und Forstsektors zuständig, andererseits arbeite ich für das European Topic Center of Climate Change Mitigation, wo ich ein kleines Team leite, das sich mit der Qualitätssicherung von Treibhausgasprojektionen der EU-Mitgliedsländer beschäftigt.

Die Haupttätigkeiten in diesem Beruf sind, große Datenmengen zu bearbeiten und Berichte zu schreiben. Dies geschieht sehr oft auch in Kooperation mit anderen (internationalen) Partnern.

\section{Was hat dir UBRM dafür gebracht?}

Alles, aber das Wichtigste ist das fachübergreifende Denken, denn Klimaschutz ist durch und durch ein interdisziplinäres Thema. Das Studium lehrt aber auch wichtige grundlegende Methoden, die man im Beruf sehr gut anwenden kann. Das wirtschaftliche Grundwissen hat sich bisher immer als sehr nützlich dargestellt.

\section{Was empfiehlst du UBRM-Studierenden?}

Macht verschiedene Praktika in unterschiedlichen Unternehmen und Institutionen, um herauszufinden, was ihr später in eurem Beruf machen wollt, v.a. was ihr nicht machen wollt! Das ist besonders für diejenigen empfehlenswert, die überhaupt nicht wissen, wohin es gehen soll. Wählt bei der Masterarbeit ein Thema, das euch auch beruflich interessiert.

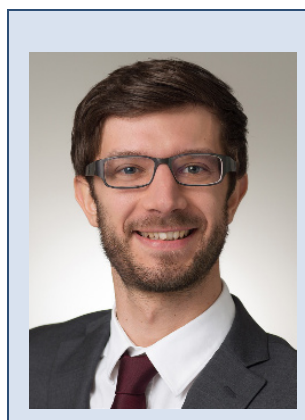

\section{Dipl.-Ing. Thomas Eberhard, BSc}

UBRM-Bachelor und -Master (Abschluss 2018, Dipl.-Ing.)

Aktuelle Tätigkeit: Junior Expert, AustriaTech GmbH (Team Automatisierte und Saubere Mobilität)

\section{Warum UBRM? (Was ist UBRM für dich?)}

Ich habe mich in der Zeit nach der Matura intensiv mit der Frage auseinandergesetzt, wo meine Interessensgebiete liegen und in welchem Bereich ich später arbeiten möchte. Als ich auf das Studium UBRM gestoßen bin, wusste ich, dass ich fündig geworden bin. UBRM ist für mich ein Studium, mit dem man sich identifizieren 
kann. Es werden umweltrelevante Aspekte nicht nur aufgezeigt und vermittelt, sondern es werden auch Lösungsansätze entwickelt. Und eben das macht die Stärke von UBRM aus: Umweltprobleme erkennen und gleichzeitig sektorübergreifende Lösungen erarbeiten.

\section{Was machst du in deinem Job?}

Meine Tätigkeiten bei AustriaTech sind in der Elektromobilität angesiedelt und umfassen ein laufendes Monitoring rund um aktuelle Trends und Entwicklungen im Bereich Elektromobilität sowie die Mitarbeit in verschiedenen Fachgremien und Arbeitsausschüssen. Da AustriaTech eine 100\%ige Tochter des Bundesministeriums für Verkehr, Technologie und Innovation ist, liegen meine Aufgaben auch in der Unterstützung des Ministeriums bei laufenden Agenden wie Förderungen, Forschungsprogrammen und Leitfäden rund um das Thema Elektromobilität.

\section{Was hat dir UBRM dafür gebracht?}

In meiner jetzigen Arbeit bin ich in unterschiedlichen Themenbereichen tätig, von Energie und Verkehr über Gesetze und Verordnungen bis hin zu wissenschaftlichem Arbeiten ist einiges dabei. Nachdem UBRM ein Generalistenstudium ist, habe ich mir Wissen aus unterschiedlichen Disziplinen aneignen können, wovon ich in meiner Arbeit sehr profitiere. Sei es beim Netzwerken bei Fachtagungen, bei Projektmeetings oder im täglichen Austausch mit Kolleginnen und Kollegen, als UBRM-Absolvent kann ich in vielen Bereichen mitdiskutieren und meine Expertise einbringen.

\section{Was empfiehlst du UBRM-Studierenden?}

Nutzt die Möglichkeit und sammelt Erfahrungen im Ausland. Davon profitiert ihr sowohl sprachlich als auch hinsichtlich Lebenslauf und Bewerbungen. Genießt die Zeit an der BOKU, sie ist wirklich eine tolle Uni, und genießt die Zeit im Türkenschanzpark. Nutzt das vielseitige Angebot und besucht v.a. im Bachelor unterschiedliche Lehrveranstaltungen, um eure Interessen herauszufinden und euch zu orientieren. Und zu guter Letzt, feiert und erfreut euch an den netten Leuten der BOKU.

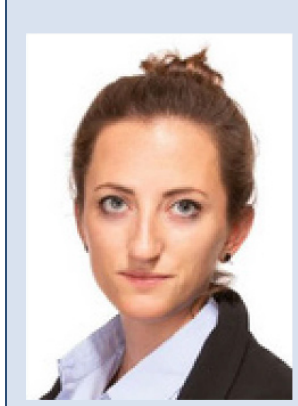

Dipl.-Ing. Annemarie Hofer, BSc

UBRM-Bachelor und -Master (Abschluss 2018, Dipl.-Ing.)

Aktuelle Tätigkeit: Consultant für Legal Compliance im Bereich Umweltrecht und Schutzrecht für Arbeitnehmerinnen und Arbeitnehmer, denkstatt (Unternehmensberatung im Nachhaltigkeitsbereich)

\section{Was ist UBRM für dich?}

UBRM ist für mich ein sehr vielseitiges Studium, in dem ich gelernt habe, inter- und transdisziplinär zu denken und zu handeln. Durch UBRM habe ich ein naturwissenschaftlich-technisches, aber auch sozialwissenschaftliches Grundverständnis in unterschiedlichen Fachbereichen erworben. Dadurch kann ich komplexe Prozesse, die gerade beim Umweltund Klimaschutz eine große Rolle spielen, besser verstehen und Probleme aus unterschiedlichen Perspektiven betrachten.

\section{Was machst du in deinem Job?}

In meinem Job versuche ich Unternehmen zu erklären, welche umwelt- und arbeitnehmerschutzrechtlichen Anforderungen sie einhalten müssen. Unser Ziel ist es, die teilweise sehr kompliziert formulierten Rechtsvorschriften für Unternehmen verständlich aufzubereiten. Neben den inhaltlichen Aufgaben bin ich auch für die Koordination unseres Legal-Compliance-Teams verantwortlich und 
übernehme unterschiedliche organisatorische Aufgaben. Zusätzlich betreue ich das interne Managementsystem der denkstatt.

In meinem Job bin ich viel unterwegs - selbstverständlich hauptsächlich mit der Bahn. Ich beschäftige mich intensiv mit unterschiedlichen Gesetzestexten und Normen, bin aber auch viel bei Kundinnen und Kunden vor Ort. Da wir auf Projektbasis arbeiten, ist der Workload unterschiedlich verteilt. Einen „klassischen Arbeitsalltag“ gibt es bei mir deshalb eigentlich nicht.

\section{Was hat dir UBRM dafür gebracht?}

Durch UBRM habe ich viele verschiedene Disziplinen kennengelernt. Prozesstechnik, Umweltsoziologie, Agrarwissenschaft, Meteorologie oder Betriebswirtschaftslehre sind nur einige davon. Dadurch habe ich auch das jeweilige grundlegende Fachvokabular gelernt. Deshalb kann ich heute mit Vertreterinnen und Vertretern unterschiedlichster Disziplinen in ihrer "Sprache" kommunizieren.

\section{Was empfiehlst du UBRM-Studierenden?}

Stellt euch darauf ein, viele unterschiedliche Dinge zu lernen, von denen ihr nicht immer wisst, ob ihr sie wirklich jemals brauchen werdet. Ihr werdet später für jede einzelne Vorlesung dankbar sein, und sei es nur, weil sie euren Horizont erweitert hat. Sucht euch Praktika und schnuppert in verschiedene Berufsfelder und Aufgabenbereiche hinein. Ich empfehle euch aber auch, euch ein Thema zu suchen, in dem ihr euch besonders gut auskennen wollt. Die Interdisziplinarität von UBRM ist einerseits wunderbar, andererseits auch die größte Herausforderung des gesamten Studiums.

\footnotetext{
Dipl.-Ing. Anton Ettl, BSc
UBRM-Bachelor und -Master (Abschluss 2014, Dipl.-Ing.)
Aktuelle Tätigkeit: Angestellter bei einem Energieversorgungsunternehmen
Was ist UBRM für dich?
Dieser Studiengang - v.a. das Bachelorstudium - ist für mich weniger eine
Berufsausbildung als eine Denkschule. Es zeigt die wesentlichsten Problem-
stellungen unserer Gesellschaft auf lokaler, nationaler und globaler Ebene auf
und versucht Antworten, bereitzustellen bzw. Studentinnen und Studenten
} 


\section{Einblicke in die Studien- \& Berufspraxis}

\section{Was hat dir UBRM dafür gebracht?}

V.a. die Spezialisierung im Masterstudium hat mir wesentliches spezifisches, technisches Wissen vermittelt. Darüber hinaus eignet man sich während des Studiums natürlich eine Vielzahl von Skills und Fertigkeiten an, die man im beruflichen Alltag zur Anwendung bringen kann.

\section{Was empfiehlst du UBRM-Studierenden?}

Allem voran natürlich das Studium zu genießen. Studierende sollten die Möglichkeiten ausschöpfen, die einem eine hervorragende Bildungsinstitution wie die BOKU bietet. Nach dem Abschluss, und darin besteht meine Hoffnung, sollte der Geist dieses einzigartigen Studiums möglichst aufrechterhalten werden.

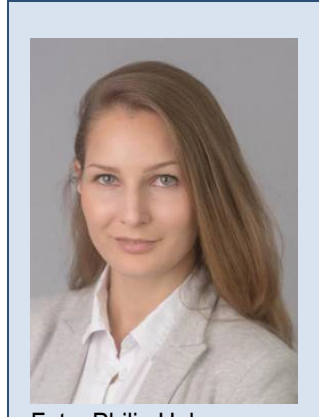

Foto: Philip Hahn

\section{Dipl.-Ing. Kathrin Höfferer, BEd BSc}

Bachelor in Agrarpädagogik (2016, BEd), UBRM-Bachelor und -Master (Abschluss 2016, Dipl.-Ing.)

Aktuelle Tätigkeit: Referentin für Energie- und Umweltpolitik, Austropapier (Fachverband der österreichischen Papier- und Zellstoffindustrie)

\section{Was ist UBRM für dich?}

Grundsätzlich sehe ich das Studium als Bindeglied der Schwerpunkte Technik, Wirtschafts- und Naturwissenschaften. Somit lässt sich UBRM auch am besten durch seine breite Fachverteilung charakterisieren, die anfangs recht willkürlich erscheint, aber sich mit fortschreitendem Studium und v.a. beim Jobeinstieg zum Vorteil entwickelt. Poetisch ausgedrückt: UBRM ist die Nuancierung seines noch nicht wahrgenommenen Ziels. Das Studium vermittelt das Werkzeug, mit dem Lösungen erarbeitet werden können - ohne einschlägigen Schwerpunkt. Klingt unspezifisch - ist es auch. Genau das bringt seinen Absolventinnen und Absolventen aber den wesentlichen Vorteil der Generalistin/des Generalisten.

\section{Was machst du in deinem Job?}

Ich arbeite als Referentin für Energie- und Umweltpolitik für den Fachverband der österreichischen Papier- und Zellstoffindustrie. Meine Position ist in diesem Bereich die Schnittstelle zwischen Wirtschaft und Politik, sowohl auf nationaler als auch auf EU-Ebene. In enger Zusammenarbeit mit der Wirtschaftskammer vertrete ich die Interessen der Papierbranche. Ich bin verantwortlich für mehrere Ausschüsse, analysiere, erarbeite und präsentiere einschlägige Fachthemen zu Energie und Umwelt.

\section{Was hat dir UBRM dafür gebracht?}

Wesentlichstes Element ist sicher das fachübergreifende Denken und der Blick über den Tellerrand. Natürlich auch die Basis in einigen technischen und wirtschaftlichen Bereichen, allerdings war mir dabei wichtig, mich durch Wahlfächer in Teilbereichen zu vertiefen.

\section{Was empfiehlst du UBRM-Studierenden?}

Praktika, Sprachweiterbildung und Zusatzqualifikationen sind Elemente, die aus keiner Bewerbung wegzudenken sind. UBRM bietet ein Programm an diversen Fortbildungen, Auslandsstudien und -reisen, wodurch die Möglichkeit geboten wird, sich in seinen Interessensgebieten zu spezialisieren. Außerdem läuft man ohne entsprechenden Einschlag nach dem Studium Gefahr, in diversen Praktika und ohne konkretem Job zu enden. Auch ein zweites Studium, das sich an eure Interessen und an UBRM anlehnt, ist schwerstens zu empfehlen. 


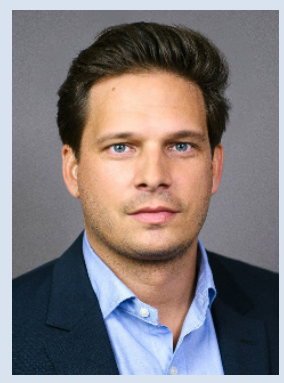

\section{Dipl.-Ing. Clemens Nießner, BSc}

UBRM-Bachelor und -Master (Abschluss 2013, Dipl.-Ing.)

Aktuelle Tätigkeit: Projektmanager, Österreichische Post AG

\section{Was ist UBRM für dich?}

Für mich ist UBRM, wie für viele der Absolventinnen und Absolventen, die Kombination aus Natur-, Wirtschafts- und Ingenieurwissenschaften. Meiner Meinung nach ist das eine ganz besondere Basis für den weiteren Berufsund Lebensweg. Man kann im UBRM-Studium vielfältige Qualifikationen erlernen. Danach stehen dann viele Türen offen - auch manche, an die man vorerst vielleicht gar nicht gedacht hat!

\section{Was machst du in deinem Job?}

In meinem alltäglichen Berufsleben beschäftige ich mich vorwiegend mit Projektmanagement. Dabei konzentriere ich mich auf klassische Projektmanagementaufgaben: von der Projektplanung, über die Projektsteuerung und die Projektkontrolle bis hin zu einem hoffentlich erfolgreichen Projektabschluss. V.a. in der Logistik und bei einem großen Logistikunternehmen wie der Post ist das eine sehr spannende und vielfältige Aufgabe!

\section{Was hat dir UBRM dafür gebracht?}

Durch das UBRM-Studium habe ich gelernt, ganzheitlich - im Sinne von verknüpft und interdisziplinär - zu denken und Sachen aus den verschiedensten Blickwinkeln zu betrachten. Im Projektmanagement sind das wertvolle Eigenschaften, da eine ganzheitliche Denkweise besonders wichtig ist, um den Überblick zu bewahren.

\section{Was empfiehlst du UBRM-Studierenden?}

Auch wenn es manchmal mühsam erscheint, eine Prüfung nach der anderen zu schreiben - kämpft euch durch das Bachelorstudium. Im Master empfehle ich euch aber, den Fokus auf ein Fachgebiet zu legen und dementsprechend auch nur eine Spezialisierung zu wählen.

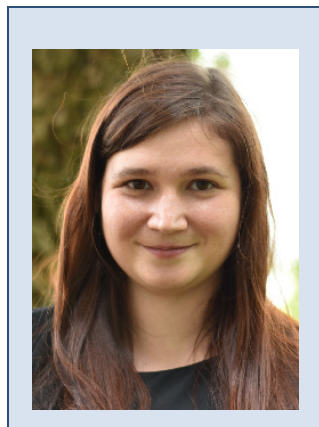

\section{Julia Zeilinger, MSc BSc}

UBRM-Bachelor (Abschluss 2014, BSc), Master in Community Water and Sanitation, Cranfield University, UK (Abschluss 2016, MSc), UBRM-Master (laufend), Doctorate School Transitions to Sustainability (laufend)

Aktuelle Tätigkeit: Wissenschaftliche Mitarbeiterin und Doktorandin am Institut für Abfallwirtschaft, BOKU

\section{Was ist UBRM für dich?}

UBRM ist für mich ein vielfältiges und zukunftsfähiges Studium, das seine Stärken in der inhaltlichen Breite und durch den Fokus auf die Schnittstellen zwischen Umwelt, Gesellschaft und Wirtschaft hat. Durch diese starke interdisziplinäre Ausrichtung ermöglicht das Studium ein Auseinandersetzen mit verschiedenen fachlichen Blickwinkeln und stellt wertvolle Werkzeuge zur Verfügung, die für die komplexen Problemfelder unserer Zeit mehr und mehr erforderlich werden. 


\section{Was machst du in deinem Job?}

Erstkontakt zum Institut für Abfallwirtschaft an der BOKU hatte ich 2011 im Zuge einer bundeslandweiten Restmüllsortieranalyse, bei der mehrere Tonnen Restmüll durch die Hände unseres kleinen Teams von Forscherinnen und Forschern gegangen sind. Seither bin ich mit einer längeren Unterbrechung aufgrund von Auslandsaufenthalten am Institut für Abfallwirtschaft angestellt - zuerst als studentische Mitarbeiterin und seit 2018 als wissenschaftliche Projektmitarbeiterin. Im Zuge eines Grundlagenforschungsprojektes zum Thema nachhaltige temporäre Wohnformen im urbanen Bereich habe ich auch die Möglichkeit, mein Doktorat zu machen. In diesem interdisziplinären Projekt, das in Kooperation von fünf BOKU-Instituten und zwei weiteren Forschungseinrichtungen durchgeführt wird, forsche ich im Bereich Abfall-, Wasser- und Ressourcenmanagement und arbeite an Life-Cycle-Assessments von temporären Wohnformen. Ich kann hier fortsetzen, was ich schon in meinen Bachelorund Masterstudien sehr geschätzt habe: Jeden Tag etwas Neues zu lernen, sich intensiv mit wissenschaftlichen Fragestellungen zu befassen und komplexen Problemstellungen auf den Grund zu gehen.

\section{Was hat dir UBRM dafür gebracht?}

Im UBRM-Studium habe ich gelernt, offen zu sein und gut zuzuhören. Mitunter sprechen unterschiedliche Fachrichtungen vom Gleichen, verwenden dafür aber ganz unterschiedliche (Fach-)Sprachen. Nachdem ich in einem sehr interdisziplinären Forschungsteam arbeite, kommt es mir zugute, grundsätzlich über verschiedene Fachgebiete Bescheid zu wissen und mitunter auch als Verbindungsglied zwischen verschiedenen Kolleginnen und Kollegen zu agieren und „Übersetzungsarbeit“ zu leisten. Zudem schätze ich den regen Austausch und den kooperativen Charakter des Studierens und Arbeitens an der BOKU.

Ich habe es immer als Bereicherung empfunden, aus so vielen Wissensgebieten lernen zu können. Bei den vielen UBRM-Lehrveranstaltungen ist es nur natürlich, dass nicht alles gleich interessant wirkt. Im Laufe der Jahre habe ich aber gemerkt, dass auch weniger spannende Lehrinhalte oftmals später sehr nützlich waren, wenn man ihnen wieder in dem einen oder anderen Kontext begegnet und sofort Anknüpfungspunkte herstellen kann.

\section{Was empfiehlst du UBRM-Studierenden?}

Geht offen durch eure Studierendenjahre und sucht euch Initiativen, in denen ihr mitgestalten könnt. Engagiert euch für etwas, das euch begeistert und am Herzen liegt. Gestaltet eure Studienzeit bzw. Lehrveranstaltungen mit, indem ihr Fragen stellt, euch in Diskussionen einbringt und euch kritisch mit den Inhalten auseinandersetzt. Studieren ist so viel mehr als die Suche nach dem nächsten Fragenkatalog und den „prüfungsrelevanten Inhalten“. Für mich war es auch immer spannend, Lehrveranstaltungen abseits des UBRM-Studienplans zu besuchen, besonders solche mit Praxisbezug, Exkursionen oder Kleingruppen. Und hebt euch am besten nicht alle vermeintlichen „Angstprüfungen“ bis zum Schluss auf.

An einigen wissenschaftlichen Konferenzen ist eine Teilnahme für Studierende gratis oder zu sehr reduzierten Teilnahmegebühren möglich. Solche Veranstaltungen ermöglichen nicht nur einen spannenden Überblick über die neueste Forschung im jeweiligen Bereich, sondern bieten auch vortreffliche Möglichkeiten zum Networking, und möglicherweise ergeben sich ja auf diesem Weg Kontakte für Praktika, Sommerjobs, Themen für Masterarbeiten oder Ähnliches.

Ich würde meine Zeit im Ausland nicht missen wollen. Im Zuge eines Double Degrees der BOKU und der Cranfield University habe ich über das Erasmus-Programm einen Master in England gemacht. Das war eine sehr bereichernde Zeit für mich. Speziell für Personen, die sich eine berufliche Zukunft in der Wissenschaft vorstellen können, kann es eine wichtige Erfahrung sein, auch andere Universitätssysteme und universitäre „Unternehmenskulturen“ kennenzulernen und internationale Netzwerke aufzubauen. 


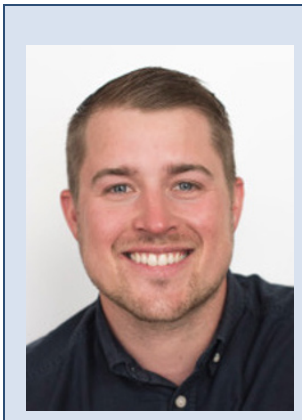

\section{Dipl.-Ing. David Tanner, BSc}

UBRM-Bachelor und -Master (Abschluss 2016, Dipl.-Ing.)

Aktuelle Tätigkeit: Junior Business Developer, RP Global Austria GmbH (im Bereich Wasser-, Wind- und Solarkraftwerke)

\section{Was ist UBRM für dich?}

UBRM ist für mich die optimale Querschnittsmaterie der Bereiche Technik, Wirtschaft und Ökologie. In diesem Studium wird ein ganzheitliches Denken vermittelt und Lösungsansätze bereitgestellt, um umweltpolitische Problemstellungen sowohl auf regionaler, nationaler als auch auf globaler Ebene behandeln zu können.

\section{Was machst du in deinem Job?}

In meiner Tätigkeit bin ich zuständig für die Erforschung relevanter Daten zu neuen Technologien im Bereich der erneuerbaren Energien, zur Validierung neuer Geschäftsmöglichkeiten sowie für die Unterstützung in der internationalen Projektentwicklung.

\section{Was hat dir UBRM dafür gebracht?}

Aus dem UBRM-Studium habe ich in erster Linie die Fähigkeit, vernetzt zu denken und Aufgaben/ Projekte durch ein fundiertes Basiswissen vielseitig anzugehen, mitgenommen. Darüber hinaus hat mir die fachspezifische Vertiefung im Masterstudium in vielen beruflichen Themenfeldern weitergeholfen.

\section{Was empfiehlst du UBRM-Studierenden?}

Ich empfehle allen Studierenden, die sich für UBRM entscheiden, die umfangreiche Bandbreite im Bachelorstudium zu nutzen, um sich der eigenen Interessen und Fähigkeiten bewusst zu werden, und sich nicht von der Vielschichtigkeit abschrecken zu lassen. Ganz wichtig ist meiner Meinung nach auch, sei es durch Praktika oder Nebenjobs Berufserfahrung in den zukünftigen Branchen zu sammeln, die in der Wahl der Spezialisierung im Master ausschlaggebend sein können. Während des Studiums Auslandserfahrungen durch die diversen Angebote (Erasmus etc.) sammeln zu können, ist eine einmalige Chance, die sehr zu empfehlen ist.

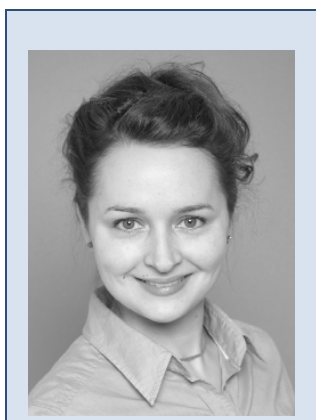

\section{DDipl.-Ing. Julia Schilder}

Wirtschaftsingenieurwesen (Maschinenbau) (Abschluss 2011, Dipl.-Ing.), UBRM-Master (Abschluss 2017, Dipl.-Ing.)

Aktuelle Tätigkeit: Programme Budget Officer, UN-Sekretariat in New York

\section{Was ist UBRM für dich?}

Vor meinem UBRM-Master habe ich Wirtschaftsingenieurwesen studiert und kenne daher die rein betriebswirtschaftliche Herangehensweise, bei der externe Kosten von wirtschaftlichen Aktivitäten zulasten der Umwelt und/oder Gesellschaft nicht betrachtet werden. Wir spüren heute, wie sich dieses Vorgehen mit rasanter Geschwindigkeit auf unseren Lebensraum Erde auswirkt. UBRM ist meiner Meinung nach eine Studienrichtung, die es dringend braucht - v.a. in Bezug auf die Umsetzung von Nachhaltigkeit in Ökologie, Ökonomie und Sozialem durch Politik, Wirtschaft und die Gesellschaft. 


\section{Was machst du in deinem Job?}

Aktuell bin ich im UN-Sekretariat in New York im Bereich Policy-Beratung für die Budgetplanung (5 Mrd. US\$, ausgenommen Peacekeeping) und Analyse der finanziellen Kennzahlen tätig. So habe ich die vielfältigen Bereiche der Vereinten Nationen kennengelernt und bin in die Vorbereitung der Budgetplanung für 2020 zur Vorlage in der Generalversammlung im September und in die Aufbereitung der Erklärungen zu budgetrelevanten Fragestellungen aus den zuständigen Komitees eng eingebunden. Besonders spannend ist auch, dass gerade ein neues System zur Budgetplanung eingeführt wird, gleichzeitig mit dem Wechsel von einem zweijährigen auf einen einjährigen Budgetzyklus.

\section{Was hat dir UBRM dafür gebracht?}

Die UN ist die Organisation, in der die (politischen) Anliegen der Weltgemeinschaft aufeinanderprallen. Teils habe ich mich an Kurse wie "Wissenschaft in Politik und Gesellschaft" sowie "Governance Nachhaltiger Entwicklung“ zurückerinnert - häufig geht die Spannweite der Interessen von kleinen Inselstaaten bis hin zu den „Weltmächten“ jedoch über den meist europäischen Kontext der Kurse hinaus und führt zu vergleichbaren paradoxen Situationen. Ein hoher Spezialisierungsgrad der einzelnen UN-Bereiche macht eine umfassende Nachhaltigkeitsanalyse je nach Problemstellung hinreichend komplex, sodass UBRM-Absolventinnen und -Absolventen optimale Voraussetzungen haben, um mit verschiedenen Stakeholdern an einer umfassenden Strategie zu arbeiten (Big Picture).

\section{Was empfiehlst du UBRM-Studierenden?}

Das Studium ist dazu da, sich auszuprobieren - sei es, Vorlesungen aus anderen Bereichen zu belegen oder ein Praktikum bzw. einen Auslandsaufenthalt während des Studiums einzuplanen. Diese Erfahrungen sind unersetzbar - neben einer Menge Spaß bringen sie einen fachlich und v.a. persönlich viel weiter. Ergreift Chancen und bleibt neugierig in jeder Hinsicht, denn fragen kostet nichts!

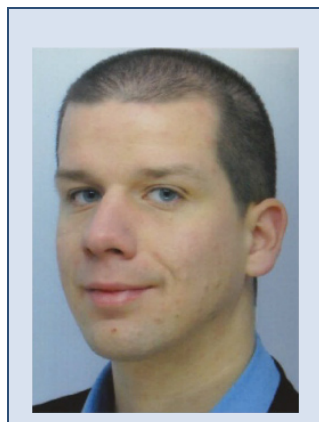

\section{Dipl.-Ing. Martin Borries, BSc}

UBRM-Bachelor und -Master (Abschluss 2011, Dipl.-Ing.)

Aktuelle Tätigkeit: Logistics \& Recycling Spezialist, Fiat Chrysler Automobiles

\section{Was ist UBRM für dich?}

UBRM ist ein breit angelegter Studiengang, der neben naturwissenschaftlichen Grundlagen auch ökonomische und soziale Themen behandelt. UBRM ist für mich ein solides Fundament zum weiteren Wissenserwerb insbesondere spezieller Thematiken im Umweltbereich. Der eher allgemeine Charakter mag anfangs etwas verwirren, nach dem Studium bemerkt man allerdings, wie gut das eigene vernetzte (interdisziplinäre) Denken funktioniert, was einen umfassenden Blick in die verschiedensten Bereiche des Wirtschaftens ermöglicht. Das Wissensspektrum reicht vom grundsätzlichen Verständnis der Stoffkreisläufe über das (Aus-)Wirken der Politik bis hin zur Funktionsweise von Wirtschafts- und Finanzprozessen.

\section{Was machst du in deinem Job?}

Mein Job als Logistics \& Recycling Specialist bei Fiat Chrysler Automobiles in Frankfurt am Main umfasst neben Tätigkeiten aus dem Bereich Umweltmanagement, wie z.B. die Einhaltung umweltgesetzlicher Anforderung des Unternehmens, auch administrative Aufgaben wie die Erstellung, Auswertung und Dokumentation von Datengrundlagen und Vorgehensweisen sowie vertragliche Angelegenheiten und Kostenkontrolle. Meine Position ist eine Querschnittsfunktion. Dabei spielen v.a. die 
Fachbereiche Abfallwirtschaft (z.B. VerpackV, ELV, BattG), Energiemanagement (Energiemanagementsysteme und Auditierungen) und Logistik (Lagerungs-, Lieferungs- und Rücknahmeprozesse von Fahrzeugen und Ersatzteilen) eine Rolle.

\section{Was hat dir UBRM dafür gebracht?}

UBRM hat es mir ermöglicht, Problemstellungen aus unterschiedlichen Perspektiven zu betrachten. So ist es möglich, sich mit Vertreterinnen und Vertretern der Wirtschaftswissenschaften, Ingenieurinnen und Ingenieuren, Technikerinnen und Technikern wie auch Geisteswissenschaftlerinnen und -wissenschaftlern auf Augenhöhe auszutauschen, die teilweise komplexen Problemstellungen zu verstehen und lösungsorientiert zu handeln. Das Prinzip des nachhaltigen Wirtschaftens hat sich in allen Lebensbereichen als erfolgreich erwiesen.

\section{Was empfiehlst du UBRM-Studierenden?}

Ich empfehle allen UBRM-Studierenden, sich anfangs nicht von der Bandbreite des Studiengangs abschrecken zu lassen. Gerade in diesem weit gefassten Spektrum liegt der Vorteil von UBRM. Das entbindet natürlich nicht von selbstständiger Vertiefung der eigenen Interessensgebiete. Zusätzliche Wahlfächer auch aus Masterprogrammen oder bei bevorzugten Professorinnen und Professoren sind aus meiner Sicht Pflicht im UBRM-Studium. Ergänzend empfehle ich, viele Praktika aus möglichst unterschiedlichen Bereichen/Branchen zur eigenen Interessensfindung beizumischen. Aus eigener Erfahrung kann ich sagen, dass wirklich jedes Fach im UBRM eine Bereicherung für die Ausbildung der eigenen Fähigkeiten war, auch wenn es manchmal schwierig war, sich das einzugestehen. Aus meiner Sicht ist das Entscheidende am Studieren die Bestimmung der eigenen Interessen und die Entwicklung des eigenen Charakters. Hierbei kann UBRM bei der Orientierung helfen. Die eigentliche Aktion muss aber stets aus Eigeninitiative erfolgen. Ich wünsche allen Studierenden viel Spaß im Studium und hoffe gemeinsam auf eine lebenswerte Zukunft.

\subsection{UBRM-Alumni - das Netzwerk für Absolventinnen und Absolventen}

Der Verein der Absolventinnen und Absolventen der Studien für Umwelt- und Bioressourcenmanagement, kurz UBRM-Alumni, vertritt Studierende sowie Absolventinnen und Absolventen der gleichnamigen Bachelor- und Masterstudien. Im Jahr 2014 gegründet, bietet der junge Verein seinen Mitgliedern in Zusammenarbeit mit dem Alumni-Dachverband der BOKU eine Vielzahl an Services an.

Der Begriff Alumni bezeichnet die Absolventinnen und Absolventen einer Hochschule oder Institutionen des tertiären Bildungsbereiches.

Die Hauptaufgaben und Ziele des UBRM-Alumni sind es, die Verbindung der Alumni zur Universität bzw. zum eigenen Studium aufrechtzuerhalten sowie die einzelnen Alumni miteinander zu vernetzen. In regelmäßigen Netzwerktreffen berichten ausgewählte UBRM-Alumni über ihren derzeitigen Job und Werdegang, und die Alumni tauschen sich untereinander aus. 


\section{Einblicke in die Studien- \& Berufspraxis}

In regelmäßigen Newslettern informiert das UBRM-Alumni-Vorstandsteam die Mitglieder über UBRM-relevante Informationen zu Jobs, Veranstaltungen und Initiativen. UBRM-Alumni ist auf Facebook mit einer eigenen Seite, einer exklusiven Gruppe nur für Alumni-Mitglieder sowie einem LinkedIn-Profil vertreten.

Das UBRM-Alumni-Vorstandsteam nutzt verschiedene Möglichkeiten und unterschiedliche Wege, um das Studium am Arbeitsmarkt bekannter zu machen sowie Arbeitgeberinnen und Arbeitgeber über die Qualifikationen und Stärken der UBRMAlumni zu informieren.

Werde Mitglied beim UBRM-Alumni: Anmeldung beim BOKU-AlumniDachverband (auch Studierende sind willkommen).

\section{Kontakt UBRM-Alumni}

Webseite:

https://boku.ac.at/ubrm-alumni/

E-Mail:

ubrm-alumni@boku.ac.at

Facebook-Seite:

https://www.facebook.com/ubrm.alumni

Facebook-Gruppe (nur für Mitglieder):

https://www.facebook.com/groups/192660231374805/

Linkedln:

https://www.linkedin.com/in/ubrm-alumni/

\section{Literatur}

BOKU (Universität für Bodenkultur Wien) (2017): AbsolventInnenbefragung KOAB: Aggregierte Analyse der Jahrgänge 2010/11 bis 2013/14. Verfügbar in: https://short.boku.ac.at/koabergebnis [Abfrage am 27.6.2019].

BOKU (Universität für Bodenkultur Wien) (2019): AbsolventInnenstudien an der BOKU. Verfügbar in: https://short.boku.ac.at/absstudien.html [Abfrage am 27.6.2019].

Statistik Austria (2018): Klassifikationsdatenbank. Verfügbar in: http://www.statistik.at/web de/klassifikationen/klassifikationsdatenbank/ [Abfrage am 27.6.2019]. 
Open Access Dieses Kapitel wird unter der Creative Commons Namensnennung - Nicht kommerziell 4.0 International Lizenz (http://creativecommons.org/licenses/by-nc/4.0/deed.de)veröffentlicht, welche die nicht-kommerzielle Nutzung, Vervielfältigung, Bearbeitung, Verbreitung und Wiedergabe in jeglichem Medium und Format erlaubt, sofern Sie den/die ursprünglichen Autor(en) und die Quelle ordnungsgemäß nennen, einen Link zur Creative Commons Lizenz beifügen und angeben, ob Änderungen vorgenommen wurden.

Die in diesem Kapitel enthaltenen Bilder und sonstiges Drittmaterial unterliegen ebenfalls der genannten Creative Commons Lizenz, sofern sich aus der Abbildungslegende nichts anderes ergibt. Sofern das betreffende Material nicht unter der genannten Creative Commons Lizenz steht und die betreffende Handlung nicht nach gesetzlichen Vorschriften erlaubt ist, ist auch für die oben aufgeführten nicht-kommerziellen Weiterverwendungen des Materials die Einwilligung des jeweiligen Rechteinhabers einzuholen.

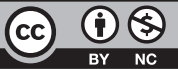

